\title{
REPRESENTACIONES SOCIALES DE ESTUDIANTES UNIVERSITARIOS SOBRE EL PLAGIO EN LA ESCRITURA ACADÉMICA
}

\author{
SOCIAL REPRESENTATIONS ABOUT PLAGIARISM IN ACADEMIC WRITING AMONG UNIVERSITY \\ STUDENTS
}

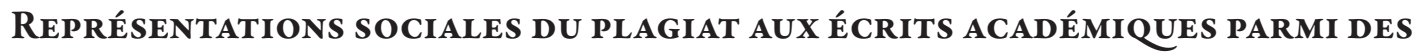 \\ ÉTUDIANTS UNIVERSITAIRES
}

\begin{abstract}
Karen Shirley López-Gil
Psicóloga, fonoaudióloga, magíster en Lingüística y Español, Universidad del Valle. Máster en Innovación e Investigación en Educación y estudiante del doctorado en Educación, ambos en la Universidad Nacional de Educación a Distancia (UNED), España. Docente e investigadora, Departamento de Comunicación y Lenguaje, y directora del Centro de Escritura Javeriano, Pontificia Universidad Javeriana, seccional Cali, Colombia.

karens@javerianacali.edu.co

http://orcid.

org/0000-0001-9826-0799
\end{abstract}

\section{María Cristina Fernández-López}

Licenciada en Español y Literatura, Universidad del Cauca. Magíster en Lingüística y Español, Universidad del Valle, Cali, Colombia. Docente del Departamento de Comunicación y Lenguaje, y coordinadora académica, Centro de Escritura Javeriano, Pontificia Universidad Javeriana, seccional Cali, Colombia.

maria.fernandez@javerianacali.edu.co https://orcid.

org/0000-0003-0296-1027

Este artículo presenta resultados parciales de la investigación "Representaciones sociales de estudiantes universitarios

\section{RESUMEN}

En este artículo se describen las representaciones sociales de los estudiantes universitarios acerca del plagio en la escritura académica. Se planteó un enfoque mixto de investigación, con un diseño descriptivo transversal. En los instrumentos de recolección de información se incluyó una encuesta en línea, aplicada a 414 estudiantes, y se llevaron a cabo dos grupos de discusión, con ocho participantes cada uno. Se usó la técnica de análisis de contenido con apoyo del software Atlas. Ti 8.0. Los resultados se agruparon en cuatro categorías: conocimientos sobre el plagio, actitudes hacia el plagio, interacción con el contexto académico y prácticas de uso de información. Sobre el conocimiento, se encontró que los estudiantes identifican el concepto de plagio y sus principales características. Respecto a las actitudes, los alumnos perciben el plagio como una práctica inadecuada y deshonesta que debe evitarse, y que les genera temor y bloqueos en la escritura. En cuanto a la interacción, la universidad demanda una mayor rigurosidad en el uso de la información que la educación secundaria, pero ofrece pocas orientaciones. Para evitar el plagio, en sus prácticas de escritura, los estudiantes usan el parafraseo y atribuyen la autoría a partir de la indicación de los enlaces de las páginas web consultadas. Aunque los participantes reconocen el plagio como una acción incorrecta, afirman que, en general, no cuentan con las competencias necesarias para evitarlo en sus textos académicos.

Palabras clave: plagio; escritura académica; escritura en la universidad; alfabetización académica; representaciones sociales.

\section{Abstract}

The purpose of the research was to identify the social representations that university students have about plagiarism in academic writing. A mixed research approach was proposed, with a cross-sectional descriptive design. The data collection instruments were an online survey applied to 414 students, and two discussion groups, with eight participants each. The content analysis technique was used, with the support of Atlas.Ti 8.0 software. The results were grouped into four categories: knowledge about plagiarism, attitudes towards plagiarism, interaction with the academic context and practices of information use. On knowledge, it was found that students identify the concept of plagiarism and its main characteristics. Regarding
\end{abstract}

Received: 2017-12-26 / Accepted: 2018/11/07 / Published: 2019/01/01

DOI: $10.17533 /$ udea.ikala.v24n01a06 
sobre el uso de información digital en la escritura de textos académicos", aprobada en la convocatoria Capital Semilla (2016) de la Pontificia Universidad Javeriana, Cali, código 00004452 attitudes, students conceive plagiarism as an inadequate and dishonest practice that should be avoided and that generates fear and blockages in writing. About interaction, the university demands a greater rigor in the use of information than secondary education, but offers few guidelines. To avoid plagiarism, in their writing practices, students use paraphrasing and attribute authorship based on the indication of the links of the web pages consulted. Although the participants recognize plagiarism as an incorrect action, they affirm that they do not have the necessary competences to avoid it in their academic texts.

Keywords: plagiarism; academic writing; university; social representations; digital sources of information.

\section{RÉSUMÉ}

Le but de la recherche était d'identifier les représentations sociales que les étudiants ont sur le plagiat dans l'écriture académique. Une approche de recherche mixte a été proposée, avec un design descriptif transversal. Dans les instruments de collecte d'informations, un sondage en ligne a été inclus, appliqué à 414 étudiants, et deux groupes de discussion ont été organisés, avec huit participants chacun. La technique d'analyse de contenu a été utilisée, avec le support du logiciel Atlas.Ti 8.0. Les résultats ont été regroupés en quatre catégories : connaissances sur le plagiat, attitudes envers le plagiat, interaction avec le contexte académique et pratiques d'utilisation de l'information. Sur la connaissance, il a été constaté que les étudiants identifient le concept de plagiat et ses principales caractéristiques. En ce qui concerne les attitudes, les étudiants conçoivent le plagiat comme une pratique abusive et malhonnête à éviter et qui génère la peur et les verrouille par écrit. En ce qui concerne l'interaction, l'université exige une plus grande rigueur dans l'utilisation de l'information que l'enseignement secondaire, mais offre peu de lignes directrices. Pour éviter le plagiat, dans leurs pratiques d'écriture, les étudiants utilisent la paraphrase et attribuent la paternité en fonction de l'indication des liens des pages web consultées. Bien que les participants reconnaissent le plagiat comme une action incorrecte, ils affirment qu'ils n’ont pas les compétences nécessaires pour l'éviter dans leurs textes académiques.

Mots clés : plagiat ; écriture académique ; université ; représentations sociales ; sources d'information en ligne. 


\section{Introducción}

La escritura en la universidad es producto de la búsqueda, el análisis, la selección y el uso de información especializada. Además del conocimiento sobre el tema, sobre la lengua y sobre el proceso de escritura, el estudiante debe saber integrar la información de referencia, utilizar mecanismos de citación, construir sentido alrededor de las citas seleccionadas y profundizar en un análisis que reúna todos estos elementos para presentar un texto que dé cuenta de su saber (Ochoa y Cueva, 2014, p. 98).

En los niveles educativos anteriores a la universidad, la escritura de los alumnos es más cercana a la reproducción del discurso del profesor y de los libros de texto (Bazerman, 2014). En la universidad, se encuentran con la necesidad de establecer diálogos con distintos autores y con la exigencia de construir una voz propia en sus escritos, proceso que, en general, resulta desafiante para los alumnos (Carlino, 2005).

Con el desarrollo de las tecnologías de la información y la comunicación, principalmente con el acceso casi ilimitado a múltiples fuentes, se han transformado los modos de relacionarse con la información. Si bien las tecnologías hacen importantes aportes a las nuevas prácticas de escritura, también pueden representar algunos riesgos. Uno de estos riesgos es la reproducción indiscriminada de ideas, que se ha convertido en un grave problema para la educación superior (Comas y Sureda, 2007; Egaña, 2012).

Este fenómeno ha sido denominado por distintos autores como "ciberplagio" (Sureda, Comas y Urbina, 2005; Sureda y Comas, 2009), para destacar que el uso incorrecto tiene una estrecha relación con el medio digital. El acceso rápido y la obtención de datos con pocos clics llevan a que haya un mayor porcentaje de plagio de las fuentes digitales que de las fuentes impresas (Jaramillo y Rincón, 2014; Šprajc, Urh, Jerebi, Trivan y Jereb, 2017). A esto se suma la concepción generalizada de que aquello que circula en la red tiene una naturaleza pública. Prácticas vernáculas digitales como el remix $x^{1}$ y los fenómenos virales de comunicación se pueden estar trasladando al ámbito académico (Cassany, 2013). De igual manera, las características del hipertexto, marcado por la vinculación de información a través de enlaces, pueden aumentar la confusión de los estudiantes respecto a la identificación de las autorías (Santana, 2008).

Cuando un estudiante reproduce las ideas de otro, sin atribuir los créditos correspondientes, además de incurrir en un comportamiento poco ético, está desaprovechando el potencial epistémico de la escritura, pues pierde la oportunidad de transformar el conocimiento a partir del diálogo con otros referentes culturales (Bereiter y Scardamalia, 1992). Este hecho puede afectar directamente el proceso formativo, debido a que no se construyen los fundamentos conceptuales de la disciplina. Skaar (2015) se refiere a esta situación como un proceso de seudoescritura, pues, aunque hay un producto escrito, este no logra las características necesarias para llegar a la función epistémica.

Por otro lado, si bien la idea básica de lo que significa el plagio está bastante extendida, pueden no ser claras para los estudiantes las implicaciones que tiene, ni cuáles son las estrategias para evitarlo (Anney y Mosha, 2015; Castro, Yoplac-López, Carpio-Tello, Sihuay-Torres y Cósar-Quiroz, 2018). Esta es una constante en la educación superior, en donde existe la advertencia y la sanción, pero no siempre se ofrecen las orientaciones acerca de los mecanismos para enfrentar este problema.

Con este panorama, resulta necesario para la academia identificar los elementos que influyen en el uso incorrecto de la información, en aras de plantear propuestas que fortalezcan los procesos

1 El remix es un concepto retomado del campo musical y se usa en los Nuevos Estudios de Literacidad para referirse a la mezcla o combinación creativa de artefactos culturales en cualquier modalidad de representación de información (Cassany, 2013, p. 106). 
formativos. Particularmente, esta investigación busca identificar las representaciones que tienen los estudiantes sobre el plagio, bajo la premisa de que las representaciones sociales guían las prácticas (Wells, 2001) y su conocimiento permite una mayor comprensión del fenómeno del plagio en el contexto universitario. Nos preguntamos, entonces, ¿cuáles son las representaciones sobre el plagio que tienen los estudiantes cuando escriben textos académicos? Específicamente, ¿cuáles son sus conocimientos y actitudes respecto al plagio y cómo se relacionan estos aspectos con las exigencias propias del contexto universitario?

\section{Algunos antecedentes}

El plagio es un fenómeno que se ha presentado desde la Antigüedad. Personajes icónicos y respetados como Platón, Aristóteles, Virgilio, para mencionar solo algunos, fueron señalados de haber copiado las ideas de otros (Sanabria, 2014, p. 90). En la era de la información digital, el fenómeno se ha hecho más visible. Diferentes estudios demuestran que la facilidad para encontrar información en la web y luego "pegarla" en los propios textos es una de las principales razones de la ocurrencia del plagio (Baird y Dooey, 2014; Šprajc et al., 2017). Sin embargo, la mayoría de autores coinciden en que el plagio es un fenómeno multicausal, en el que intervienen factores como la motivación, la orientación vocacional, la sobrecarga académica, la ética individual, los valores del grupo social, las orientaciones de los docentes y las formas de evaluación en las instituciones.

Los valores culturales, las dinámicas sociales modernas, la globalización, la inmediatez, el deseo de figurar para los otros, la sensación de poder acceder a todo lo que se desea a través de los espacios virtuales, entre otros factores, han causado profundos cambios en la comunicación y por ese mismo camino en las formas de leer y de escribir (Ruiz, 2016, p. 219). La pérdida del sentido de lo que es original, la sensación de que todo es de todos y de que nada es perdurable, favorece la idea de que la información pertenece a ese cúmulo de objetos que habitan el ciberespacio y que pueden ser retomados sin permiso (Linne, 2014).

Muchas de las investigaciones que presentan resultados sobre plagio se han derivado de estudios más amplios sobre fraude, generalmente, desde perspectivas de desarrollo moral o desde la línea de integridad académica, esta última relacionada con el estudio de los principios éticos que permiten a los sujetos actuar de acuerdo con los valores de las comunidades académicas, atendiendo a factores sociales e individuales (Santos et al., 2017, p. 759). Turrens, Staik, Curtis y Burling (2001, como se citó en Ordóñez, Mejía y Castellanos, 2006, p. 37) indican que los estudiantes universitarios de Estados Unidos pueden incurrir en conductas de fraude en un rango entre 75 y $98 \%$. Agud (2014) sostiene que, en las universidades españolas, hasta un $94 \%$ de los estudiantes reconoce haber tenido algún comportamiento fraudulento. En lo que respecta particularmente al plagio académico, Comas, Sureda y Oliver (2011) hicieron un estudio con 1025 universitarios españoles, de los cuales el 69,2\% admite haber copiado textos, $70 \%$ afirma "reciclar" sus propios textos (autoplagio), 82,9\% indica que no suele citar tablas ni gráficos, 19,5\% nunca incluye las referencias de consulta de internet y 34,6\% dice que lo hace muy poco. También en el contexto español, Egaña (2012) analiza el plagio en estudiantes universitarios y encuentra que, ante una actividad práctica, el 45,2\% de los participantes no interpreta de forma adecuada una referencia.

Un aspecto interesante en estas investigaciones ha sido la constatación de que el plagio no siempre es un comportamiento deliberado y que no en todas las ocasiones se debe a problemas de orden ético o moral de los estudiantes (Comas et al., 2011, p. 378). Una de las líneas que se ha derivado de estos estudios se ha centrado en comprender el fenómeno del plagio desde las creencias, las percepciones y los conocimientos de los involucrados, y desde su relación con las competencias informacionales y de lectura y escritura académicas (Ochoa y Cueva, 2014; 2016). 
La manera como se enseña en algunas asignaturas y el tipo de demandas que se hacen pueden favorecer la presencia de plagio, ya que muchas de las tareas que los alumnos enfrentan en la universidad "estimulan más la repetición que la creatividad" (Santos et al., 2017, p. 757) y su propósito no siempre es claro para los estudiantes.

La evaluación es otro factor de gran relevancia. Evaluaciones de tipo sumativo, en las que se valora solo el producto y no se hace un seguimiento del proceso, tienen una mayor incidencia en las prácticas de plagio (Martínez, Borjas y Andrade, 2015, p. 4).

\section{Fundamentación teórica}

Tres elementos esenciales guiaron esta investigación. Por un lado, la propuesta de la alfabetización académica que delimita el campo de estudio, debido a que el plagio es un fenómeno que se aborda desde distintas disciplinas e implica el análisis desde perspectivas legales, éticas, psicológicas, sociológicas, etc. Por otro lado, la noción de "plagio" y, finalmente, la noción de "representación social”, por ser el aspecto del plagio en que se quería centrar el análisis.

Esta propuesta retoma la perspectiva de alfabetización académica, que aborda específicamente las características de la lectura y de la escritura en la universidad. Aunque se inició en países anglosajones varias décadas atrás, desde hace un poco más de 15 años ha tenido un importante impacto en la fundamentación de investigaciones y en el desarrollo de programas en instituciones de América Latina (Carlino, 2013). La alfabetización académica se refiere a la manera como las instituciones favorecen la incorporación de los estudiantes a la comunidad científica y profesional, mediante la apropiación de los modos de leer y de escribir de sus disciplinas.

Un postulado esencial de este enfoque es que las demandas de la escritura en la universidad difieren considerablemente de las que se realizan en niveles educativos anteriores. Al respecto, Carlino (2003) plantea que en la universidad se espera que los estudiantes sean autónomos en la búsqueda de información, mientras que en la educación secundaria los docentes esperan que los estudiantes retomen lo que se les ha transmitido. En la educación superior, se anhela también que los estudiantes trasciendan la comprensión e interpretación de los textos y puedan asumir posturas críticas frente a lo que leen, mientras que en la secundaria se promueven más las prácticas de reproducción del conocimiento. Otra diferencia importante entre estos niveles educativos es que en la universidad se aspira a que los estudiantes reconozcan que el conocimiento tiene autores y diferentes miradas, mientras que en la escuela parece asumirse que hay un solo y verdadero conocimiento.

Un elemento que destaca de la escritura académica, aunque no es exclusivo del contexto académico, es la intertextualidad, que se refiere a las relaciones que se establecen entre el texto propio y otros referentes culturales (Bazerman, 2013). El uso de las ideas de otros autores evidencia que el estudiante se está apropiando de los conocimientos de su área disciplinar y permite dar mayor solidez a sus propios planteamientos. Para esto, aquel debe familiarizarse tanto con las formas de dialogar con otras voces, como con las normas que le permiten evidenciar estos diálogos y atribuir los créditos correspondientes. De esta manera, la intertextualidad se concreta a través de mecanismos de citación.

Cuando estas normas no se siguen, es decir, cuando hay un uso inadecuado de la información, los estudiantes incurren en "plagio". Este es un término difícil de definir, pues puede ser entendido de múltiples formas y referirse a una amplia gama de prácticas. Particularmente, en la legislación colombiana no figura el término "plagio" (Rojas y Olarte, 2010, p. 537), pero sí se presentan más de 250 artículos relacionados con los derechos de autor. La ley de derechos de autor entra en vigor para la mayoría de los países europeos a partir de la Convención de Berna en 1886, y para América 
Latina, en donde toma el nombre de "propiedad intelectual”, luego de la Convención de Montevideo en 1889.

La expresión "propiedad intelectual” se define en la legislación colombiana como "una disciplina normativa que protege las creaciones intelectuales provenientes de un esfuerzo, trabajo o destreza humanos, dignos de reconocimiento jurídico" (Rengifo, 1996, p. 21). El artículo 2 de la Ley 23 de 1982 del código civil colombiano afirma que

Los derechos de autor recaen sobre las obras científicas, literarias y artísticas [...] cualquiera que sea el modo o forma de expresión y cualquiera que sea su destinación, tales como: los libros, folletos y otros escritos [...] (Colombia, Congreso de la República, 1982).

Dicho esto, esta ley protege la forma de expresar las ideas por escrito, mas no la idea en sí misma. Así las cosas, queda claro que en el texto emergente es permitido retomar la idea original y elaborar una interpretación de dicha idea, siempre que se manifieste de manera diferente. Esto, de acuerdo con la norma, vela por el interés público que posee toda sociedad de replicar, ampliar o refutar criterios en aras del desarrollo científico y cultural de una comunidad; pero cuando el uso es inadecuado, puede constituir un ilícito civil o, incluso, un delito penal (Toller, 2011, p. 86).

El plagio en la escritura se refiere, entonces, al uso de la información retomada de otros textos, sin atribuir de modo explícito y correcto los créditos de autoría. Puede darse de manera deliberada, cuando el autor es consciente de que está tomando la información de otro y decide no otorgar los créditos; pero puede darse también de forma involuntaria o por desconocimiento, cuando el escritor no tiene suficiente dominio de los mecanismos de citación. Al respecto, Childers y Bruton (2016) afirman que la mayoría de estudiantes reconoce como inadecuada la práctica de copiar textualmente fragmentos de otros escritos sin indicar el autor, pero pocas veces identifican que un parafraseo muy parecido al original o que retomar las ideas con las propias palabras sin atribuir la autoría también son modalidades de plagio.

Finalmente, en este estudio se indaga por las representaciones sociales de los estudiantes sobre el plagio académico. El interés por estudiar las representaciones surge, principalmente, por su incidencia sobre los comportamientos de los sujetos, pues la representación social es una forma de conocimiento práctico que se expresa en lo cotidiano; su principal función es establecer un orden que les permita a los individuos orientarse en el mundo que los rodea, al tiempo que contribuye a la construcción social de la realidad (Wells, 2001). En este sentido, Carlino (2006) sostiene que las representaciones inciden de modo efectivo en las prácticas y las guían hacia ciertas acciones, en tanto esfuerzos de comprender el mundo social, estabilizarlo y homogeneizarlo.

La noción de "representación social" tiene como antecesor el concepto de representación colectiva, acuñado por Durkheim en 1898. Moscovici retomó este concepto en 1961, definiendo la representación como una forma particular de conocimiento que se genera en la interacción con otros sujetos y que permite interpretar los acontecimientos y las situaciones que viven las personas. Jodelet (1999) y Wells (2001) afirman, además, que las representaciones constituyen categorías que les permiten a las personas clasificar las circunstancias, fenómenos e individuos con quienes se relacionan.

Otra característica de las representaciones sociales es que son dinámicas o cambiantes. Esto quiere decir que no se construyen de una vez y para siempre, y que tienen la posibilidad de ser transformadas. Esta característica es muy interesante en el ámbito educativo, pues en este se busca comprender las representaciones para poder reorientar las acciones formativas y generar cambios de comportamiento en los estudiantes (Pievi y Echaverry, 2005).

Atendiendo a la revisión de los antecedentes de investigaciones sobre el plagio, se identificó que las prácticas de plagio trascienden los aspectos 
individuales. $\mathrm{Al}$ respecto, la noción de "representación social” resulta particularmente importante, debido a que esta se configura a partir de la relación de distintas dimensiones y en el marco de un contexto sociohistórico particular. Una de las dimensiones de la representación es el conocimiento o información que, de acuerdo con Moscovici (1961), se refiere a la cantidad y la calidad de los datos, las razones y las explicaciones que sustentan la representación. Otra de las dimensiones es la actitud, entendida como la reacción emocional positiva o negativa frente a una situación. $\mathrm{Al}$ ser sociales, y no meramente cognitivas, las representaciones tienen una dimensión de interacción con el contexto social en donde se articulan los conocimientos, las actitudes, las opiniones, las imágenes y las creencias (Araya Umaña, 2002, p. 39). Al estudiar las representaciones sociales es preciso indagar por cada uno de estos elementos.

\section{Metodología}

Esta investigación se situó desde un enfoque mixto, basado tanto en la cuantificación, como en la observación y la descripción del fenómeno. El diseño fue no experimental de tipo descriptivo transversal, pues se llevó a cabo sin la manipulación deliberada de variables (McMillan y Schumacher, 2005).

Participaron 414 estudiantes, matriculados en una asignatura introductoria de escritura en una universidad privada de la ciudad de Cali, Colombia. Aunque la asignatura se dirige a ingresantes de distintas carreras de Ingeniería y de las carreras de Comunicación y Diseño, puede ser tomada como electiva por estudiantes de semestres superiores y de otras carreras. Así, la población estuvo constituida en un $79,7 \%$ por ingresantes y en un 20,3\% por estudiantes de semestres avanzados. La mayoría de estudiantes pertenecía a carreras de ingeniería (62\%) y en menor proporción a carreras de humanidades $(21 \%)$, ciencias experimentales $(10 \%)$ y ciencias administrativas (7\%). El $40 \%$ de los participantes fueron mujeres y el $60 \%$ hombres, con un promedio de edad de 17,5, en un rango entre 15 y 30 años.
Como instrumentos de recolección de información se usaron la encuesta en línea (Páramo y Arango, 2008) y el grupo de discusión (Ibáñez, 1986). La encuesta en linea buscaba identificar las representaciones y prácticas de los estudiantes sobre el uso de información digital en sus textos académicos, incluido el plagio. Aunque no hay consenso sobre cómo estudiar las representaciones, estas suelen expresarse mediante enunciados verbales "descriptibles y diferenciados producidos por actores sociales como formas de percepción y simbolización de aspectos claves de la experiencia social" (Mato, 1999, como se citó en Bocca y Vasconcelo, 2008, p. 23). De esta manera, se plantearon enunciados directos, analogías y situaciones que nos permitieron acercarnos a las representaciones configuradas por los participantes.

La encuesta se administró electrónicamente a los 414 participantes e incluyó seis preguntas de caracterización de la población; ocho preguntas de opción múltiple, relacionadas con el uso de información digital; una pregunta de opción múltiple con única respuesta, en la que se debían identificar situaciones de plagio; una pregunta de valoración con escala Likert, que indagó sobre las actitudes de los estudiantes, y una pregunta abierta acerca de sus conocimientos respecto al plagio.

Este instrumento fue validado mediante el juicio de expertos y se aplicó una versión de prueba con quince sujetos similares a la población objeto de estudio. Los expertos evaluaron cinco aspectos del instrumento: la coherencia entre los ítems del instrumento y los objetivos de investigación, la coherencia entre las preguntas del instrumento, la claridad de los enunciados y las opciones de respuesta, el ajuste a la audiencia a la que se dirigía y la extensión. Por su parte, los participantes del estudio piloto evaluaron la claridad de los enunciados, el ajuste a la audiencia y la extensión. Estas valoraciones fueron positivas (en promedio, 4,75 en los distintos aspectos, en una escala de 0 a 5) y los evaluadores hicieron algunas recomendaciones de ajuste que fueron incorporadas al instrumento 
en su versión final. Estas recomendaciones estuvieron relacionadas principalmente con la claridad de los enunciados.

Por otra parte, se desarrollaron dos grupos de discusión, con ocho integrantes cada uno, en los que se profundizó en algunos de los hallazgos de la encuesta. Se asumió el planteamiento de Ibáñez (1986), quien entiende esta técnica como un mecanismo de producción de discursos organizados, guiados y estructurados por un investigador que indaga sobre un fenómeno social específico. Los participantes del grupo son invitados a conversar respecto al tema y a expresar sus conocimientos, intereses y expectativas acerca del fenómeno, lo que permite al investigador cotejar sus hipótesis o ideas previas.

La convocatoria para participar en la investigación se hizo a través de los docentes de los grupos de la asignatura, quienes a su vez compartieron la información con los estudiantes y reportaron quiénes estaban interesados en participar. La participación fue voluntaria y no implicó retribuciones económicas ni tuvo una incidencia en las calificaciones del curso.

Los grupos de discusión fueron grabados en audio y posteriormente se transcribieron los discursos producidos. A cada participante se le otorgó un código de identificación y se mantuvieron sus datos básicos (carrera, edad, semestre, sexo) para las interpretaciones.

Para analizar la información de la encuesta se usaron medidas de tendencia central y gráficos de frecuencias.

Por otra parte, se usó el análisis de contenido para los resultados de naturaleza cualitativa (respuestas abiertas del cuestionario y transcripción de los grupos de discusión), con apoyo del software Atlas.Ti 8.0.

De acuerdo con Bardin (1996), el análisis de contenido es un conjunto de técnicas que examina las comunicaciones humanas y que usa indicadores para interpretar mensajes producidos en determinados contextos sociales. El procedimiento de análisis suele incluir cinco pasos: establecer el objeto de análisis, determinar las reglas de codificación, definir el sistema de categorías, comprobar la fiabilidad del sistema de codificación-categorización y realizar inferencias sobre los hallazgos.

\section{Resultados}

Los hallazgos se agruparon en cuatro categorías. Las tres primeras - "Conocimientos", "Actitudes" e "Interacción”- corresponden a dimensiones que tradicionalmente se estudian en el campo de las representaciones sociales (Araya Umaña, 2002); la cuarta categoría - "Prácticas" - se incluyó por su estrecha relación con las representaciones y por ser un tema que emergió constantemente en los discursos de la pregunta abierta y los grupos de discusión. En cada categoría se presentan, de forma integrada, los resultados de ambos instrumentos.

\section{Conocimientos sobre el plagio}

Se refiere a la cantidad y la calidad de la información, los datos, las razones y las explicaciones que sustentan la representación, así como su origen (Moscovici, 1961). En esta categoría se agrupan los conocimientos que los estudiantes tienen sobre el plagio.

En la encuesta se indagó por el concepto de plagio en los textos académicos, mediante una pregunta abierta. En las respuestas, se evidenció que los estudiantes lo reconocen como una acción incorrecta, que consiste en tomar las ideas de otras fuentes de información sin atribuir los créditos de autoría. En la figura 1 se muestran las palabras más usadas por los estudiantes en sus definiciones (se incluyeron términos con mínimo 70 repeticiones en el total de las respuestas. El más frecuente, "ideas", tuvo 372 apariciones).

Varios estudiantes identificaron, como características del plagio, que: 1) puede ser o no intencional; 


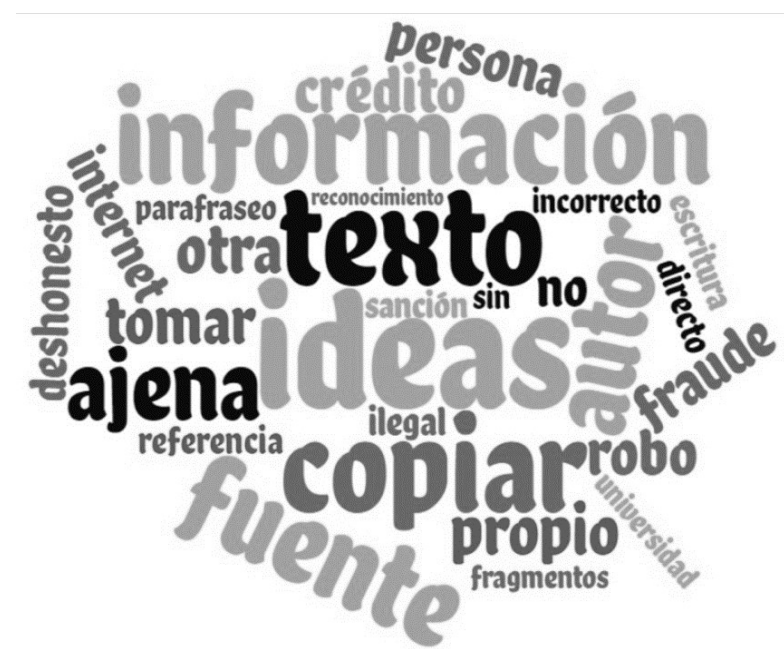

Figura 1 Nube de palabras. Análisis de contenido con Atlas.Ti 8.0

2) la información usada puede estar en distintas modalidades de representación; 3) puede estar en formato impreso o digital, aunque es más común incurrir en plagio a partir de la consulta de fuentes digitales, y 4) puede darse a través de una copia textual o de un parafraseo.

1) El plagio es uno de los errores más cometidos en la universidad, intencional o sin intención y se trata específicamente del robo de ideas de textos sin su respectiva fuente, pues la persona que incurre en esto no estaría dando el crédito al autor (RA85). ${ }^{2}$

1) El plagio es el hurto de ideas no propias en nuestros documentos, ya sea intencional o no. Se da cuando tomamos fragmentos o ideas de un texto y no aclaramos el autor ni demás datos que orienten que nuestro texto está retomando ideas de otro texto (RA341).

2) El plagio sería la acción de manipular información, ya sea textual o visual, y usarla como si fuera propia (RA21).

2 Las citas del discurso de los participantes se identifican con las letras RA y números entre 1 y 414 para indicar las respuestas abiertas de la encuesta. Con las letras GD y números entre 1 y 16 se identifican las citas de los participantes en los grupos de discusión (Grupol, participantes 1 al 8; Grupo 2, del 9 al 16). Las respuestas de los estudiantes se presentan con ajustes ortográficos, pero no sintácticos.
3) Plagio es tomar de alguna página de internet, pero también de un libro, revista, periódico o publicación frases, ideas o explicaciones como propias sin mencionar al autor legítimo (RA30).

4) El plagio no se da únicamente en la copia exacta de las palabras o citas, sino también en la adaptación y el parafraseo de ideas que no sean propias, sin indicar que se retoma de un texto ajeno (RA317).

2) 4) Situación en la que se presenta copia textual o parafraseo de un tema, puede ser de un texto, imagen, símbolo o cualquier otra actividad parecida, sin citar al autor que realizó el trabajo o darle reconocimiento por el trabajo suministrado (RA324).

En otra pregunta de la encuesta relacionada con el conocimiento sobre el plagio, los participantes debían identificar situaciones de uso incorrecto de la información. La mayoría de estudiantes reconoció como inadecuado copiar fragmentos o parafrasear sin indicar la fuente. No obstante, menos de la mitad identificó que sería un error copiar la mayor parte de un texto, aun cuando se indique la autoría (véase figura 2). Esto evidencia que, aunque los estudiantes tienen un conocimiento general sobre el plagio, desconocen algunas situaciones en las que se puede dar un uso inadecuado de la información.

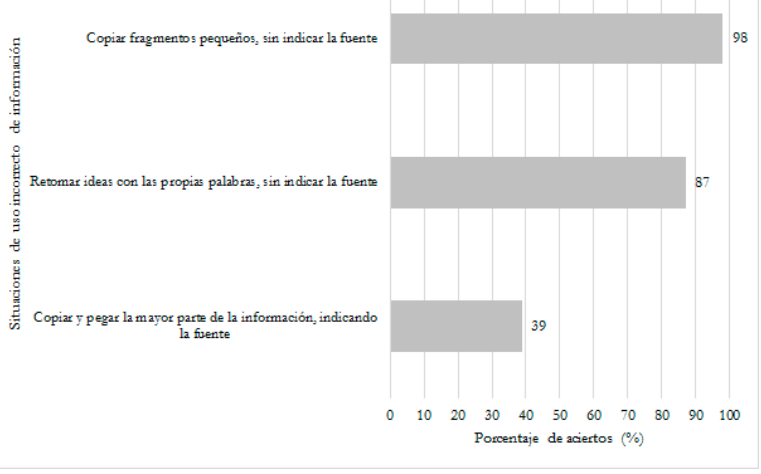

Figura 2 Identificación de situaciones de uso incorrecto de la información

\section{Actitudes respecto al plagio}

La actitud es entendida como la disposición favorable o no frente a un fenómeno o situación 
(Moscovici, 1961). Su principal función es regular las acciones vinculadas con la representación social. En esta categoría se incluyen los resultados relacionados con la disposición emocional de los estudiantes ante el plagio en la universidad.

Dos elementos llaman la atención respecto a las actitudes de los estudiantes: 1) la relación del término "plagio" con nociones como "fraude" o "robo",y el uso de algunas analogías que equiparan el plagio con el hurto de bienes materiales; 2) el temor que manifiestan ante las posibles sanciones del plagio y los bloqueos que esto genera en sus procesos de escritura. Los estudiantes temen a las consecuencias negativas, aunque no tienen mucho conocimiento sobre las sanciones. Uno de los problemas referidos por los estudiantes es que no siempre saben con certeza a qué fuente deben atribuir la autoría.

1) A mi parecer, el plagio es un delito muy grave, producto de la deshonestidad. En los trabajos académicos se evidencia el plagio, por ejemplo, en una evaluación; pero esta antiética manera de proceder es más extensa y abarca el campo del trabajo investigativo (RA152).

1) Robar ideas es como ir a un banco y robar 1000000000 de dólares, es como entrar sin permiso a la alcoba de una mujer desconocida y mirar su vida personal, cuando está desnuda (RA414).

2) A mí me da mucho temor el plagio, por todas las sanciones que implica (GD1).

2) A mí me bloquea, me demoro mucho más cuando estoy escribiendo, como 20 minutos pensando cómo incluyo cada cita (GD5).

2) Me bloqueo mucho porque no sé de dónde he obtenido toda la información que sé, hay cosas que ya sé, pero no identifico claramente de dónde viene y siempre hay que indicar cuál es el soporte. Me da mucho miedo caer en plagio (GD8).

En una pregunta de la encuesta se pidió a los estudiantes que indicaran su grado, de acuerdo con cinco afirmaciones sobre el uso de información, en una escala Likert con opciones desde "Totalmente en desacuerdo" hasta "Totalmente de acuerdo" (véase figura 3).

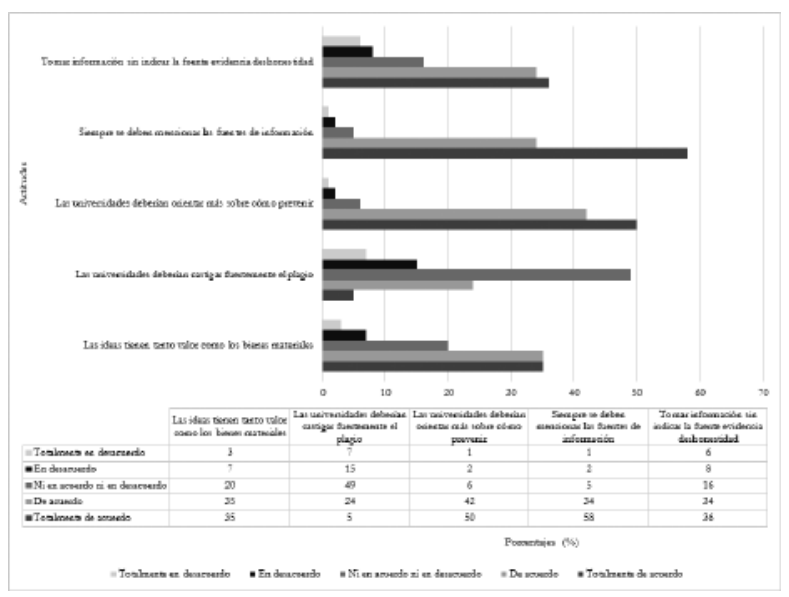

Figura 3 Actitudes sobre el uso de información en textos académicos

Los estudiantes estuvieron "Totalmente de acuerdo" y "De acuerdo" con varias afirmaciones: "No indicar la fuente es una práctica deshonesta", "Siempre se debe mencionar la fuente de donde se tomó la información", "Las ideas tienen tanto valor como los bienes materiales" y "La universidad debería brindar más orientaciones sobre cómo evitar el plagio". No obstante, más de la mitad indicó no estar ni de acuerdo ni en desacuerdo con la idea de que el plagio sea castigado fuertemente en la universidad. Esta valoración se evidenció también en los grupos de discusión:

No conozco bien las sanciones, pero creo que deberían ser menos estrictos, al menos en los primeros semestres, no sé (GD2).

Es un cambio muy grande del colegio a la universidad y uno no está preparado y puede cometer errores, no siempre de forma voluntaria, por eso es injusto que el plagio se castigue gravemente (GD9).

\section{Interacción: exigencias del contexto académico}

La interacción se refiere a las relaciones que existen entre los conocimientos y las actitudes construidos por los sujetos y las características del entorno sociocultural. Las representaciones se construyen justamente en la relación con los otros miembros de la cultura (Araya Umaña, 2002). Esta categoría incluye resultados asociados a las exigencias del contexto educativo respecto al plagio. 
En los grupos de discusión, los estudiantes destacaron que el plagio en la escritura es un concepto propio de la universidad, ya que en los niveles educativos anteriores pocas veces tenían que apoyarse en otras fuentes de información y se hacía poca mención a los mecanismos de citación. En el contexto universitario, la intertextualidad es un requisito indispensable (Soto, 2009). De acuerdo con los participantes, en el colegio el fraude se asociaba con copiar durante un examen o con alterar algún resultado.

En mi caso, en el colegio no nos pedían citas, no vimos nada sobre citación. A veces todos llegábamos con la misma información al salón, de Wikipedia. No había un criterio para citar. No sabíamos qué era el plagio. En la universidad sí hubo un choque fuerte, porque sí existen varios criterios para escribir el trabajo (GD14).

En el colegio era más cumplir, cortar y pegar. Acá en la universidad sí nos ha tocado aprender a buscar información, interpretarla y citarla correctamente (GD15).

De igual manera, los estudiantes indicaron que la universidad ejerce mucha presión, que tienen una "sensación de amenaza" de incurrir en plagio, pero que no reciben las orientaciones necesarias para evitarlo, pues los profesores dan por sentado que ya han desarrollado las competencias necesarias para trabajar con la información.

Principalmente no se enseña, es decir, hay unas sanciones y hasta te pueden dar un taller, pero es algo que debe hacerse siempre, con un criterio compartido con los profesores para que no te confundas (GD9).

Sigo sintiendo que hace falta instrucción en la aplicación. Las normas de citación son claras, pero, en ocasiones, al momento de aplicarlas uno no sabe qué hacer. Creo que el acompañamiento de los profesores debe estar ahí, en la práctica, no tanto en facilitar un manual para seguir al pie de la letra (GD16: 8).

\section{Prácticas sobre uso de información}

Las prácticas son entendidas como acciones que se repiten cotidianamente y que se mantienen en el tiempo. Evidencian la concreción de las representaciones y se construyen mediante la participación de los sujetos en la vida social, cultural y política
(Pievi y Echaverry, 2005, p. 3). Esta categoría incluye las prácticas relacionadas con la citación en la escritura académica.

Los estudiantes identificaron los mecanismos de citación como la principal estrategia para evitar el plagio:

[...] para evitarlo debes hacer bien tus citas y decir siempre de dónde tomaste la información (RA286).

Siempre que se hacen citas textuales hay que revisar bien que se hayan incluido todos los datos y si es un parafraseo hay que revisar mucho más, para que no se parezca al texto original ni a otros textos (GD3).

Los alumnos usan el parafraseo y la integración de distintas fuentes con más frecuencia que la citación directa (véase figura 4). Indican que en pocas ocasiones copian y pegan, posiblemente por la concepción de esta práctica como inadecuada o como la que puede llevar más fácilmente al plagio académico.

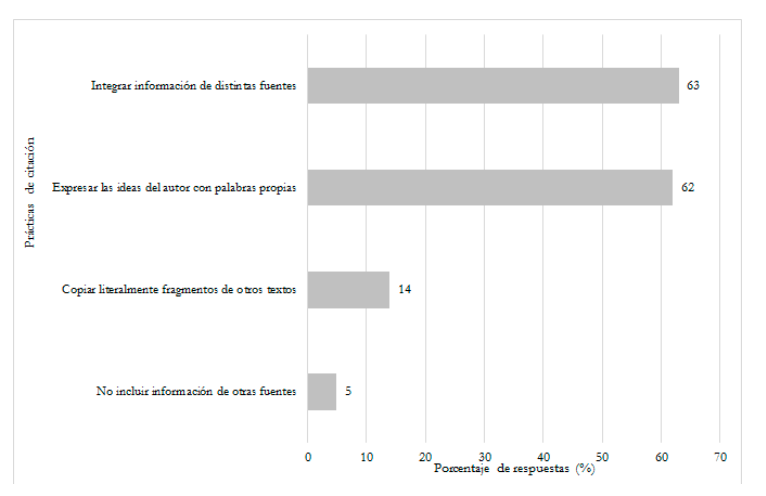

Figura 4 Prácticas de citación de los estudiantes

Respecto a la inclusión de los datos para atribuir la autoría, la mayoría de los estudiantes saben que deben explicitar las fuentes en las que se han apoyado, pero puede haber confusiones respecto a qué datos incluir (véase figura 5), pues algunos indican solo el enlace. El $95 \%$ de los participantes afirma que consulta información en la web para elaborar sus textos académicos, por lo que casi todas las referencias son de fuentes digitales. En las prácticas, no se encontraron diferencias importantes entre los estudiantes ingresantes y los estudiantes de semestres avanzados. 


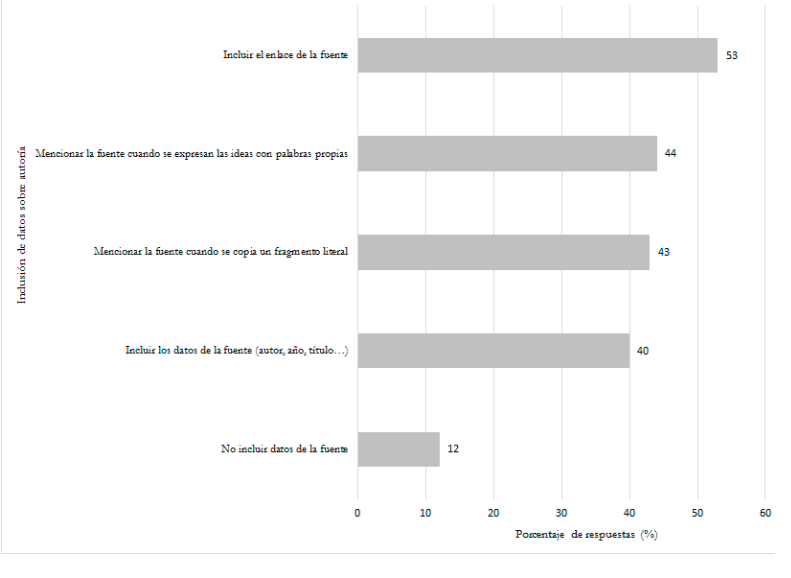

Figura 5 Inclusión de datos de la fuente

Finalmente, los estudiantes reconocen que existe abundante material sobre mecanismos de citación, pero también expresan las dificultades a las que se enfrentan cuando no reciben suficientes orientaciones por parte de los profesores:

A mí me tocaba buscar en internet, pero era confuso.

Por lo menos, a veces hacía una mezcolanza entre APA

4. ${ }^{\mathrm{a}}$ edición y la 5. ${ }^{\mathrm{a}}$, o entre APA e ICONTEC. Eso fue hasta que una profesora nos facilitó un Power Point ${ }^{\circ} \mathrm{y}$ ya tengo dónde solucionar mis dudas (GD15).

\section{Discusión}

En los principales hallazgos de esta investigación se destacó que los estudiantes reconocen las características básicas del plagio, pero no algunas de sus especificidades, ni tienen suficiente claridad sobre cuáles son sus implicaciones o los mecanismos para evitarlo. Las actitudes, en general, son desfavorables, pues se rechaza el plagio por considerarlo una práctica deshonesta; no obstante, los estudiantes no están seguros de que el plagio deba ser sancionado estrictamente. La posibilidad de incurrir en plagio incide negativamente en los procesos de escritura, genera bloqueos y temores. Los estudiantes reconocen también un importante cambio del contexto respecto a la exigencia sobre el uso de información, pues no estaban habituados a un uso riguroso de las fuentes en los niveles educativos previos a la universidad. Aunque los estudiantes usan algunas estrategias para evitar el plagio, como redactar con sus propias palabras lo que han consultado en distintas fuentes, no siempre tienen claridad acerca de cómo atribuir la autoría.

Estos resultados evidencian la necesidad de que los alumnos tengan más conocimiento sobre el plagio, considerando, además, que cada institución puede tener sus propias definiciones y normas específicas (Ortega, 2011, p. 3). Al respecto, más allá del conocimiento de las sanciones, se espera que los estudiantes puedan comprender el sentido de la atribución de la autoría y sus implicaciones éticas y, de esta manera, que el último y definitivo instrumento que guíe el quehacer académico sea "la recta conciencia, entendida como la capacidad de orientar las propias decisiones hacia lo bueno" (Tudela y Aznar, 2013, p. 24).

De igual manera, se requieren acompañamientos que permitan a los estudiantes comprender las dinámicas propias del contexto académico. Muchos de los problemas que se pueden presentar con el uso de información pueden deberse a la falta de familiaridad con los modos de leer y de escribir en la universidad (Ennam, 2017, p. 140), y este desconocimiento de los mecanismos y normas puede llevar a situaciones de plagio involuntario (Childers y Bruton, 2016). De modo similar, se requiere la facilitación de espacios de reflexión sobre la búsqueda, la selección y el uso de información, entendidos como prácticas sociales situadas en un contexto $\mathrm{y}$ una cultura específicos (Cabra-Torres, Marciales, Castañeda-Peña, Barbosa-Chacón y Melo, 2016).

Las orientaciones de los docentes, los tipos de tareas de escritura asignadas y las formas de evaluación también son elementos que se deben tomar en cuenta, pues las demandas propias del contexto académico pueden ser uno de los aspectos que más influye en que los alumnos incurran en prácticas de plagio académico (Egaña, 2012; Ochoa y Cueva, 2014). Los estudiantes afirman que, con frecuencia, se enfrentan a consignas muy distintas de los docentes o a la ausencia de explicaciones sobre cómo usar la información en los textos, lo que puede confundir y dilatar el proceso escritural. 
En este sentido, proponer una evaluación con una intencionalidad formativa y orientadora, centrada en el proceso y no solo en el producto, puede restar presiones que a la larga interfieren en la toma de decisiones en el momento de escribir (Martínez et al., 2015). Por otro lado, la formación debe incluir, también, el ilustrar a los estudiantes sobre los diferentes tipos de plagio, pues estos manifiestan una conceptualización única de un fenómeno que abarca distintas modalidades (Ortega, 2011).

En este marco, es posible concluir que la orientación en el establecimiento de relaciones de intertextualidad y, por lo tanto, la prevención del plagio, debe atravesar todo el proceso formativo. Este acompañamiento no debe agotarse en los cursos introductorios de escritura o en talleres específicos, sino que igualmente debe hacer parte de las asignaturas propias de cada disciplina, pues, como se evidenció en los resultados, es una necesidad tanto de los ingresantes como de los estudiantes de semestres superiores.

Una propuesta sería articular las asignaturas con actividades de trabajo colaborativo entre docentes disciplinares y el docente de lengua, con el objetivo de acompañar tanto al profesor como al estudiante en actividades en las que prime la apropiación no solo de los contenidos de la asignatura, sino también de las competencias retóricas necesarias para expresarse sobre dicha temática en un marco epistemológico concreto, incluyendo además las competencias informacionales. Investigaciones en este sentido demuestran el impacto de esta estrategia en la práctica de los profesores y en el aprendizaje de los estudiantes (Lledó y Perandones, 2011; López-Gil y Molina, 2018).

La pertinencia de la articulación se refuerza al escuchar a los estudiantes en los grupos de discusión, cuando expresan la ansiedad que sienten en el momento de parafrasear. En cuanto a este tema, los profesores de lengua tenemos un fuerte trabajo por hacer, pues la actividad cognitiva de retomar información y convertirla en información nueva convoca varias y complejas operaciones mentales que permiten, entre otras, la comprensión y la síntesis de los contenidos, aspectos que no necesariamente son parte de las competencias pedagógicas de los docentes disciplinares.

Otra estrategia que puede hacer carrera en el marco de la problemática en las instituciones de educación superior es abrir espacios de apoyo permanente para los estudiantes, en los cuales se aborde el tema del plagio de manera concreta, por ejemplo, desde su definición, los diferentes modos de plagio y los mecanismos que contribuyen a evitarlo, como el manejo de los formatos y estilos de citación de cada área (normas APA, IEEE, Harvard, Vancouver...) y el uso de software antiplagio (Turnitin', Safe Assign ${ }^{\oplus}$, Plagium, etc.). Esto, aunado a actividades de comprensión que faciliten el desarrollo de competencias para seleccionar y sintetizar información, operaciones necesarias en el uso de voces de autoridad en los textos académicos.

La formación debe considerar, además, la motivación de los estudiantes y hacer mayor énfasis en el pensamiento crítico (Santos et al., 2017), para, de esta manera, concientizar a los sujetos en la importancia del comportamiento ético respecto a sus trabajos académicos. Para ello, es indispensable garantizar experiencias de aprendizaje de calidad (Baird y Dooey, 2014) y hacer un uso reflexivo de las herramientas y los recursos (Heckler, Forde y Bryan, 2013).

Finalmente, la academia está llamada a atender los aspectos contextuales que pueden tener una incidencia en las prácticas que se regulan desde la universidad. Factores más amplios como las reconfiguraciones sociales y educativas, que se han dado gracias a las tecnologías, también deben ser motivo de análisis (Husain, Al-Shaibani y Ali Mahfoodh, 2017). Ruiz (2016) plantea al respecto:

\footnotetext{
[...] nos debe hacer reflexionar si la cuestión que hay tras ello es el hecho de que este tipo de trabajos académicos requiere de unas formas de lectura muy diferentes a las predominantes en la era digital y que son, en definitiva, las prácticas en las que están profundamente inmersos los estudiantes fuera de las escuelas, como apuntaba
} 
Pérez Gómez (2012) y de las aulas universitarias. Es decir, la recurrencia al plagio en los trabajos académicos plantea sobre la mesa la cuestión de si el problema estriba en que las formas de lectura que entran en juego en este tipo de prácticas universitarias están muy alejadas de esas otras formar de leer vibrantemente contemporáneas que llamaba Chartier (2010) "discontinuas, fragmentadas, segmentadas” (p. 229).

\section{Referencias}

Agud, J. (2014). Fraude y plagio en la carrera y en la profesión. Revista Clínica Española, 214(7), 410-414. DOI: http://dx.doi.org/10.1016/j.rce.2014.03.007

Anney, V., y Mosha, M. (2015). Student's plagiarism in higher learning institutions in the era of improved internet access: Case study of developing countries. Journal of Education and Practice, 6(13), 203-217.

Araya Umaña, S. (2002). Representaciones sociales: ejes teóricos para su discusión. Cuaderno de Ciencias Sociales, 27. Costa Rica: Facultad Latinoamericana de Ciencias Sociales (FLACSO). Recuperado de http:// unpan 1.un.org/intradoc/groups/public/documents/ICAP/UNPAN027076.pdf

Baird, C., y Dooey, P. (2014). Ensuring effective student support in higher education alleged plagiarism cases. Innovation in Higher Education, 39(5), 387-400. DOI: http://dx.doi.org/10.1007/s10755-014-9285-4

Bardin, L. (1996). Análisis de contenido (2. ${ }^{a}$ ed.). Madrid: Ediciones Akal S. A.

Bazerman, C. (2013). A Rhetoric of Literate Action: Literate Action. Volume 1. Perspectives on Writing. Fort Collins, Colorado: The WAC Clearinghouse and Parlor Press.

Bazerman, C. (2014). El descubrimiento de la escritura académica. En F. Navarro (Ed.), Manual de escritura para carreras de humanidades (pp. 11-16). Buenos Aires: Editorial de la Facultad de Filosofía de la Universidad de Buenos Aires.

Bereiter, M., y Scardamalia, C. (1992). Dos modelos explicativos de los procesos de composición escrita. Revista Infancia y aprendizaje, 58, 43-64.

Bocca, A., y Vasconcelo, N. (2008). Algunas reflexiones acerca de las prácticas y representaciones sociales en estudiantes universitarios: la escritura académica. Revista Enunciación, 13(1), 20-27. Recuperado de http://revistas.udistrital.edu.co/ojs/index.php/ enunc/article/download/1257/1692::pdf.

Cabra-Torres, F., Marciales, G., Castañeda-Peña, H., Barbosa-Chacón, J., y Melo, L. (2016). Competencias informacionales. Rutas de exploración en la enseñanza universitaria. Bogotá: Editorial Pontificia Universidad Javeriana.

Carlino, P. (2003). Alfabetización académica: un cambio necesario, algunas alternativas posibles. Revista Educere, 6(20), 409-420. Recuperado de http://redalyc.uaemex.mx/src/inicio/artpdfred. jsp icve $=35662008$ \&icvenum $=2061$

Carlino, P. (2005). Escribir, leer y aprender en la universidad: una introducción a la alfabetización académica. Buenos Aires: Fondo de Cultura Económica.

Carlino, P. (2006). Concepciones y formas de enseñar escritura académica. Signo \& Seña, (16), 71-117.

Carlino, P. (2013). Alfabetización académica diez años después. Revista Mexicana de Investigación Educativa, 18(57), 355-381.

Cassany, D. (2013). En_linea. Leery escribir en la red. Barcelona, España: Anagrama.

Castro, Y., Yoplac-López, B., Carpio-Tello, A., Sihuay-Torres, K., y Cósar-Quiroz, J. (2018). Percepción del plagio académico en estudiantes de Odontología. Educación Médica, 19(3), 141-145. DOI: http:// dx.doi.org/10.1016/j.edumed.2017.03.003

Childers, D., y Bruton, S. (2016). "Should it be considered plagiarism?" Student perceptions of complex citation issues. Journal of Academic Ethics, 14(1), 1-17. DOI: http://dx.doi.org/10.1007/s10805-015-9250-6

Comas, R., Sureda, J., y Oliver, M. (2011). Prácticas de citación y plagio académico en la elaboración textual del alumnado universitario. Teoria de la Educación en la Sociedad de la Información, 12(1), 359-385.

Comas, R., y Sureda, J. (2007). Ciber-plagio académico. Una aproximación al estado de los conocimientos. Revista TEXTOS de la CiberSociedad, (10). Recuperado de https://www.researchgate.net/ publication/52009644_Ciber-Plagio_Academico_Una_aproximacion_al_estado_de_los_conocimientos

Colombia, Congreso de la República (1982). Ley 23 del 28 de enero de 1982 sobre derechos de autor. Bogotá: Congreso de la República de Colombia. Recuperado de http://derechodeautor.gov.co/documents/10181/182597/23.pdf/ a97b8750-8451-4529-ab87-bb82160dd226

Egaña, T. (2012). Uso de bibliografía y plagio académico entre los estudiantes universitarios. RUSC. Universities and Knowledge Society Journal, 9(2), 18-30. Recuperado de http://www.redalyc.org/ 
articulo.oa?id=78023425003, DoI: http://dx.doi. org/10.7238/rusc.v9i2.1209

Ennam, A. (2017). Systematic analysis if the effects of digital plagiarism on scientific research: Investigating the Moroccan context- Ibn Tofail University as case study. Journal of Education and Practice, 8(2), 133-141. Recuperado de https://files.eric.ed.gov/ fulltext/EJ1131798.pdf

Heckler, N., Forde, D., y Bryan, H. (2013). Using writing assignment designs to mitigate plagiarism. Teaching Sociology, 41(1), 94-105. DoI: http://dx.doi. org/10.1177/0092055X12461471

Husain, F., Al-Shaibani, G., y Ali Mahfoodh, O. (2017). Journal of Academic Ethics, 15(2), 167-195. DOI: http://dx.doi.org/10.1007/s10805-017-9274-1

Ibáñez, J. (1986). Más allá de la sociología. El grupo de discusión: técnica y critica. Madrid: Siglo XXI.

Jaramillo, S., y Rincón, N. (2014). Los estudiantes universitarios y la sociedad de la información: una combinación que ha facilitado el plagio académico en las aulas colombianas. Información, cultura y sociedad, (30), 127-137.

Jodelet, D. (1999), La representación social: fenómenos, concepto y teoría. En: S. Moscovici (Ed.), Psicología social II (pp. 469-495). Barcelona: Paidós.

Linne, J. (2014). "Ahora hago fast food académico". Los estudiantes de la Universidad de Buenos Aires en tiempos de internet. Palabra Clave, 17(3), 695-716. DOI: http://dx.doi.org/10.5294/pacla.2014.17.3.7

Lledó, A., y Perandones, T. (2011). Hacia una cultura colaborativa entre el profesorado universitario. Revisión y fundamentación teórica del trabajo colaborativo. En: M. Gómez y J. Álvarez (Coords.), El trabajo colaborativo como indicador de calidad en el Espacio Europeo de Educación Superior (pp. 125-138). Alcoy, Alicante: Editorial Marfil, Universidad de Alicante.

López-Gil, K., y Molina, V. (2018). Incidencia del trabajo colaborativo docente en la enseñanza y el aprendizaje de la escritura académica. Revista Electrónica de Investigación Educativa, 20(1), 1-13. Recuperado de https://doi.org/10.24320/redie.2018.20.1.1477

Martínez, A., Borjas, M., y Andrade, J. (2015). El fraude académico universitario: el caso de una universidad privada de la ciudad de Barranquilla. Zona Próxi$m a$, (23), 1-17. DoI: http://dx.doi.org/10.14482/ zp. 23.7184

McMillan, J., y Schumacher, S. (2005). Investigación educativa (5. ${ }^{\mathrm{a}}$ ed.). Madrid: Pearson.
Moscovici, S. (1961). El psicoanálisis, su imagen y su público. Buenos Aires: Huemul.

Ochoa, L., y Cueva, A. (2014). El plagio y su relación con los procesos de escritura académica. Forma y Función, 27(2), 95-113.

Ochoa, L., y Cueva, A. (2016). Percepciones de estudiantes acerca del plagio: datos cualitativos. $R e-$ vista Encuentros, 14(2), 25-41. DoI: http://dx.doi. org/10.15665/re.v14i2.822

Ordóñez, C., Mejía,J., y Castellanos, S. (2006). Percepciones estudiantiles sobre el fraude académico: hallazgos y reflexiones pedagógicas. Revista de Estudios Sociales, (23), 37-44.

Ortega, E. (2011). La respuesta al plagio en la educación superior: un estudio internacional. Interdisciplinaridad y Sinergias en Economia y Negocios, XX International Congress of the European Academy of Management and Business Economics (AEDEM). Montenegro, 5-6 de septiembre de 2011. European Academics Publishers.

Páramo, P., y Arango, M. (2008). Cuestionarios. En P. Páramo (Ed.), La investigación en ciencias sociales. Técnicas de recolección de información (2. ${ }^{a}$ ed.) (pp. 55-84). Bogotá: Universidad Piloto de Colombia.

Pievi, N., y Echaverry, E. (2005). Representaciones sociales sobre la enseñanza universitaria y su resignificación en el proceso de formación docente. Memorias del Congreso Internacional en Educación, Lenguaje y Sociedad: tensiones educativas en América Latina. Universidad Nacional de La Pampa. Argentina, del 23 al 25 de noviembre de 2005. Recuperado de http://www.memoria.fahce.unlp.edu.ar/trab_eventos/ev.6766/ev.6766.pdf

Rengifo, E. (1996). Propiedad intelectual. El moderno derecho de autor. Bogotá: Universidad Externado de Colombia.

Rojas, M., y Olarte, J. (2010). Plagio en el ámbito académico. Revista Colombiana de Anestesiologia, 38(4), 537-538. Recuperado de http://www.scielo.org.co/scielo.php?script $=$ sci_arttext\&pid $=$ S0120-33472010000400010

Ruiz,A.(2016).Fuentesdigitalesyfuentesimpresas.Prácticas letradas y plagio en el contexto universitario. Revista Chilena de Literatura, (94), 215-230. Recuperado de http://www.scielo.cl/scielo.php?script=sci_artte xt\&pid=S0718-22952016000300011, DOI: http:// dx.doi.org/10.4067/S0718-22952016000300011

Sanabria, L. (2014). Conceptualización jurídica del plagio en Colombia. Revista Colombiana de Cirugia, 
29(2), 88-97. Recuperado de http://www.scielo.org. co/pdf/rcci/v29n2/v29n2a2.pdf

Santana, O. (2008). Entre o plágio e a autoria: qual o papel da universidade? Revista Brasileira de Educacao, 13(38), 357-368.

Santos, C., Dos Santo, P., Sant’Ana, M., Masuda, H., Barboza, M., y Vasconcelos, S. (2017). Going beyond academic integrity might broaden our understanding of plagiarism in science education: A perspective from a study in Brazil. Anais da Academia Brasileira de Ciencias, 89(1), 757-771. DOI: http://dx.doi. org/10.1590/0001-3765201720160474

Skaar, H. (2015). Writing and pseudo-writing for Internet-based sources: Implications for learning and assessment. Literacy UKLA, 49(2), 69-76. DOI: https://doi.org/10.1111/lit.12045

Soto, G. (2009). Intertextualidad explícita en textos académicos de estudiantes universitarios. Un estudio exploratorio. Literatura y Lingüistica, (20), 141157. Recuperado de http://www.scielo.cl/pdf/lyl/ n20/art08.pdf
Šprajc, P., Urh, M., Jerebi, J., Trivan, D., y Jereb, E. (2017). Reasons for plagiarism in higher education. Organizacija, 50(1), 33-45. DOI: http://dx.doi.org/10.1515/ orga-2017-0002.

Sureda J., Comas, R., y Urbina, S. (2005). The "copy and paste” generation: Plagiarism amongst students, a review of existing literature. International Journal of Learning, 12(2), 161-168.

Sureda, J., y Comas, R. (2009). Las causas del plagio académico entre el alumnado universitario según el profesorado. Revista Iberoamericana de Educación, 50(1), 197-220.

Toller, F. (2011). Propiedad intelectual y plagio en trabajos académicos y profesionales. Revista La Propiedad Inmaterial, (15), 85-97.

Tudela, J., y Aznar, J. (2013). ¿Publicar o morir? El fraude en la investigación y las publicaciones científicas. Revista PersonayBioética, 17(1), 12-27. Recuperadodehttp:// www.redalyc.org/articulo.oa?id=83228613002

Wells, G. (2001). Indagación dialógica. Hacia una teoría y una práctica socioculturales de la educación. Temas de educación. Barcelona: Paidós.

How to reference this article: López-Gil, K. S., y Fernández-López, M. C. (2019). Representaciones sociales de estudiantes universitarios sobre el plagio en la escritura académica. Íkala, Revista de Lenguaje y Cultura, 24(1), 119-134. Dor: 10.17533/udea.ikala.v24n01a06 